\title{
Weight and Body Image Perceptions in Nutrition and Dietetics University Students
}

\author{
Lisa Herzig $^{1} \oplus$, Jamie Levitt ${ }^{1}$, Kaitlynn Watson ${ }^{1} \oplus$, and Gretchen L. George ${ }^{2} \oplus$ \\ ${ }^{1}$ Fresno State University, Department of Food Science and Nutrition \\ ${ }^{2}$ San Francisco State University, Nutrition and Dietetics Program of the Family Interiors Nutrition \& \\ Apparel Department
}

\begin{abstract}
Background and Purpose: Stereotypical and prejudicial attitudes towards people considered overweight or obese are documented in professionals ranging from physicians, nurses, fitness and general nutrition professionals, and registered dietitian nutritionists (RDN) and may introduce barriers to equitable care. To identify the prevalence of anti-fat attitudes (AFA); fat phobia (FPS); and body appreciation scores (BA) in nutrition and dietetics' students (ND) and non-nutrition and dietetics' students (NND) through a crosssectional design. Methods: During 2018, students $(n=297)$ from two California State Universities completed a survey including three validated instruments. Additionally, height, weight, and waist circumference were collected using NHANES procedures. A series of ANCOVA's and correlation coefficients were computed. Results: Significant differences existed in BA between ND (M=3.61, $\mathrm{SD}=0.66)$ and NND students $(\mathrm{M}=3.81, \mathrm{SD}=0.70) ; \mathrm{t}(288)=2.49, \mathrm{p}=0.013$. Scores indicated existing antifat attitudes and fat phobia. Significant positive correlations existed between FPS and anthropometrics. Weight related perceptions were identified. Conclusion: A need exists for a fundamental evidence-based training specifically focused on knowledge and awareness related to health metrics and social justice pedagogy to help RDN work unbiasedly with patients of all shapes.
\end{abstract}

(C) 2021 and CC-BY 4.0 licensed by the authors.

Keywords: weight-stigma, weight-bias, body mass index, registered dietitian nutritionist, training overweight/obesity.

\section{Introduction}

Modern cultures idealize thinness and denigrate heaviness (Puhl, Latner, O’Brien, Luedicke, Danielsdottir, \& Forhan, 2015; Schwartz, O'Neal, Chambliss, Brownell, Blair, \& Billington, 2003), which can contribute to stereotypical and prejudicial attitudes towards people considered overweight or obese. This prejudice, or bias, is observed within healthcare students and in health professionals ranging from physicians, nurses, fitness and health coaches, and registered dietitian nutritionists (RDN) (Obara, Vivolo, \& Alvarenga, 2018; Panza, Armstrong, Taylor, Puhl, Livingston, \&
Pescatello, 2018; Puhl, Wharton, \& Heuer, 2009; Techman \& Brownell, 2001). Documented associations from these professions link stereotypes of being 'worthless, lazy and stupid' with obese individuals (Pearl, Wadden, \& Tronieri, 2018), resulting in adverse consequences, such as negative message internalization. This then, further prompts a patient to develop unhealthy behavioral compensations (Puhl et al., 2009). Also of concern, is the psychological barrier that may arise for clients seeking health care for prevention and/or treatment. The patient may feel uncomfortable and avoid contact if weight bias is 
sensed, further putting them at risk (Pearl \& Puhn, 2016; Pearl et. al, 2018; Phelan, Burgess, Yeazel, Hellerstedt, Griffin, \& Ryn, 2015; Puhl et al., 2009; Schvey, Puhl, \& Brownell, 2014; Wadden, \& Tronieri, 2018; Wu \& Berry, 2018). In order to help clients, become successful, they must be able to feel accepted regardless of their body size (Constance \& Sauter, 2011). While these prejudices are not new, they have become a larger issue due the persistent and increasing American obesity epidemic (Crandall, 1994; Greenhalgh, 2012; Hales, Carroll, Fryar, Ogden, 2020).

\section{Obesity Rates in the U.S.}

Approximately $42.4 \%$ of American adults (20 years and older) and $20.6 \%$ of American youth (2-19 years old) are considered obese (body mass index $\geq 30$, BMI, a ratio of weight to height, $\mathrm{kg} / \mathrm{m}^{2}$ ) (Hales et al. 2020; Hales, Fryar, Carroll, Fryar, \& Ogden, 2017; Ogden, 2018). While BMI has the limitation of an indirect assumption of fat accumulation, it is widely used in medical assessment (Andersen, Baird, Bates, Chapel, Cline, Ganesh, S.N... McCauley, 2018). Understanding fat accumulation and distribution is clinically relevant, as accretion around the waist has associations with excess subcutaneous and visceral fat, of which the latter is linked to higher comorbidity risk such as heart disease and diabetes (González-Muniesa, MártinezGonzález, \& Hu, 2017).

Currently, the RDN uses BMI to calculate nutritional needs and to assist in making nutritional diagnoses for patient care plans (Andersen et al., 2018; Ashell \& Gibson, 2016; Raynor \& Champagne, 2016; Ross, Neeland, Yamashita, Shai, Seidell, Magni, \& Després, 2020). While weight and BMI are key variables of a client's evaluation, they are not the most important and by themselves can be misleading. BMI cannot define a patient's ability to adhere to a treatment plan, explain their dietary intake, predict their self-confidence, or stereotype their overall lifestyle choices. Fitzgerald et al. stated that implicit associations, whether conscious or unconscious, can biasly influence a health care professional's judgement (FitzGerald \& Hurst, 2017). Implicit biases help to explain the possible dissociation between a healthcare professional's belief that they want to treat all patients equally versus their negative thoughts and actions.

\section{Biases among Healthcare Providers}

Further studies, with varied methodology, have found evidence of imbedded biases amongst healthcare providers (Aboueid, Pouliot, Nur, Bourgeault \&, Giroux, 2019; Panza et al., 2018; Puhl et al., 2015; Schwartz et al., 2003; Swift, Hanlon, El-Redy, Puhl, \& Glazebrook, 2013; Teachman \& Brownell, 2001). A study conducted over ten years ago evaluated mock case studies in a sample of college students majoring in nutrition and dietetics and identified moderate amounts of fat phobia, similar to the general public, and various implicit biases towards individuals categorized as overweight and/or obese (e.g. poorer diet quality, less likely to comply with treatment plan, less self-control, lower selfesteem) (Puhl et al., 2009). A 2013 randomized intervention with nutrition students (e.g. bachelor of nutrition science, masters of nutrition science) and medical students (e.g. Bachelor of Medical Science, Masters of Nursing students) from the United Kingdom identified a moderately high weight bias at baseline measurement as determined by various validated tools such as Anti-Fat-Attitude Questionnaire (AFA) and Fat Phobia Scale (FPS) (Swift et al., 2013). After the six-week anti-weight bias video viewing intervention no significant improvements were measured in implicit bias, but some improvements in explicit bias were improved (Swift, Tischler, Markham, Gunning, Glazebrook, Beer, \& Puhl, 2013). A more recent systematic review found in six different studies that RDNs believed excess weight was related to personal choice first before genetics or biology (Jung, Luck-Sikorski, Wiemers, \& Riedel-Heller, 2015). Thus, this accumulation of findings demonstrates the need for healthcare professionals to address the issue of their own implicit biases which prevent them from 
supporting patients holistically. Furthermore, steps need to be put into place that focus on removing the specific negative explicit attitudes towards patient characteristics such as obesity. The end goal would be to treat all patients equally and with respect, regardless of shape or size.

Another important observation is the relationship with mild body dysmorphia, disordered eating, and weight stigma. Studies have indicated that the prevalence of disordered eating and eating disorders to be higher in RDNs, which may contribute to altered perceptions of self and others, impacting professionalism (Bjornsson, Didie, \& Phillips, 2010; Tremelling, Sandon, Vega, \& McAdams, 2017). These observations are of concern as current Academy of Nutrition and Dietetics Accreditation Council for Education in Nutrition and Dietetics (ACEND) accreditation guidelines do not specifically outline weight bias training guidelines for undergraduate students and dietetics interns, nor do continuing education requirements.

In the Academy of Nutrition and Dietetics (The Academy) Practice Paper on Treatment of Overweight and Obesity in Adult, there is one paragraph referencing the importance of RDN's being "free of weight bias" and underscoring the essentiality of clearly understanding the etiology of obesity, yet no clear guidelines exist to support this treatment recommendations (Raynor \& Champagne, 2016). While the general RDN scope of practice training protocol follows The Academy Code of Ethics in which many of the 4 principles and 32 standards emphasize the equal treatment of individuals, weight bias and body dysmorphia are still rampant (Andersen et al., 2018; Panza et al., 2018).

Professional suggestions have been made in studies where these biases have been identified and in a recent 2019 issue of Today's Dietitian, which reaches the practicing RDN (Dennett, 2019, Panza et al., 2018; Puhl et al., 2009). It is suggested that RDNs and educators of future RDN's consider embracing Health at Every Size pedagogy, Intuitive Eating, as well as 'get comfortable with the uncomfortable', meaning the conversations about patient weight etiology if it goes beyond the usual scope (diet, exercise) and connects to societal issues (access, trauma, culture) (Bégin, Carbonneau, Gagnon-Girouard, Mongeau, Paquette, Turcotte, \& Provencher, 2019; Brown, 2009; Dennett, 2019).

\section{Food and Diet Experts}

RDN's are experts in diet practices and medical nutrition therapy, suggesting they work with many individuals impacted by this obesity epidemic to improve nutrition and health comorbidities and outcomes. Currently the ACEND undergraduate accreditation standards for nutrition and dietetics programs, known as Knowledge for the Registered Dietitian Nutrition (KRDN's) do not specifically require comprehensive education or classroom applications regarding image, weight perception, and body dysmorphia (Andersen et al., 2018). That said, weight bias exists and may impact standard of care due to misunderstanding of the diverse etiology of weight gain (e.g. psychosocial factors, food insecurity, and trauma) and more importantly resulting from a lack of comprehensive empathy training in burgeoning nutrition professionals. Researchers have indicated a need for interventions and change to the training approach of nutrition and dietetic students, but little is seen in current research (O'Brien, Puhl, Latner, Mir, \& Hunter, 2010; Puhl et al., 2009; Puhl, Latner, Mir, \& Hunter, 2010; Techman \& Brownell, 2001).

What is currently being done in the classroom may not be enough, as seen in some studies, or may not be consistent enough for a larger impact on the entirety of the newly trained nutrition and dietetics student (Panza et al., 2018; Swift et al., 2013). In a Canadian study, nutrition and dietetics students were polled about the type and amount of weight bias information they were being taught. Results highlighted a wide array of responses which correlated to amount of intervention, thus identifying inconsistency in 
perception and impact of weight bias education as well as potential for impact (Dwyer, Starr, Mills, \& Haines, 2016).

\section{The Present Study}

Considering much of the research on weight bias in the nutrition and dietetics profession in the United States has been done sparsely over the last 10 years, collecting additional information about the current prevalence is helpful to add to body of knowledge and identify and understand the magnitude of the problem. This information can be used for modifying pedagogy to support student success, defined by academic standards (e.g. GPA, internship placement) and improved future professionalism (e.g. less weight bias), in nutrition and dietetics majors and improve overall RDN practices. Furthermore, the authors believe it is important to understand body appreciation in addition to weight stigma in order to direct future educational objectives.

Therefore, the main purpose of this study was to understand the differences in 1) anti-fat attitudes; 2) fat phobia; and 3) body appreciation between two groups; a sample of nutrition and dietetics students and a sample of non-nutrition and dietetics students at two universities in the Western United States. Furthermore, two secondary objectives were to investigate the association between the participants body mass index (BMI), waist circumference (WC), waist to height ratio (WHtR) to their responses from these validated surveys.

\section{Methods}

\section{Study Design}

During 2018, college students from two universities in the Western United States participated in a cross-sectional study related to weight and body stigma.

\section{Sample}

A sample of nutrition and dietetics students (ND) from accredited Didactic Programs in Dietetics and non-nutrition and dietetics (NND) students $(n=297)$ were recruited with flyers and department emails. Interested students completed a pre-screen to determine eligibility (e.g. $>18$ years old, ND or NND) using Qualtrics methodology. Once deemed eligible, students were scheduled for a single in-person appointment. The scheduling occurred by text messaging or phone calls from a trained research assistant. When participants arrived for the appointment, they were explained the consent, and also allowed to read the consent independently and decide if they would like to participate by signing the form. The study protocol for human subject consent was approved by the respectful universities Institutional Review Boards: X IRB (X18-07) and X IRB no-risk departmental review.

\section{Measures and Data Collection}

The online survey was a combination of standard research developed demographic questions and 3 validated surveys.

Anti-Fat Attitudes Questionnaire (AFA). This is a 13-item tool that assesses individual's explicit antipathy toward fat people (e.g. 'I don't like fat people very much') and also assesses the belief that being overweight is a matter of personal control or lack thereof (e.g. 'Fat people tend to be fat pretty much through their own fault') (Crandall, 1994). Items are scored on a Likert scale $(1=$ 'very strongly disagree' to $9=$ 'very strongly agree'), summed and divided by the number of items used to create the subscale, giving a possible range between 13 and 9. In both cases higher scores indicate greater anti-fat attitudes. Previous research has demonstrated that the subscales were internally consistent (Cronbach's alpha coefficient $\geq 0.7$ ).

Fat Phobia Scale (FPS). This 14-item scale assesses fat phobia towards overweight and obese individuals (Bacon, Scheltema, \& Robinson, 2001). In this scale 14 pairs of adjectives are used to describe persons with obesity (e.g. selfindulgent vs. self-sacrificing) and using 5-point Likert scale respondents select which describes their beliefs best. Higher scores indicate a higher 
fat phobia. Previous research demonstrated that the F-scale was reliable (Cronbach's alpha coefficient $\geq 0.7$ ).

Body Appreciation Scale (BAS). A 14-item tool assessing an individual's acceptance and appreciation of their bodies (Avalon, Tylka, \& Wood-Barcalow; 2005). This tool is considered a psychometrically sound tool that measures body image and is appropriate for research and educational contexts to understand and promote body appreciation.

\section{Anthropometric}

Measurements.

Undergraduate research assistants at both universities were trained to collect height, weight, and waist circumference from participants using NHANES anthropometry collection method (National Health and Nutrition Examination Survey [NHANES] Anthropometry Procedures Manual, 2017). Each University has a lab space where these measurements were collected using privacy screens. The SECA high capacity digital flat scale was used for each body weight measurement. The SECA wall mount stadiometer was used to measure the participant's height. Lastly, the waist circumference measurements were taken using SECA 201 tape measures waist circumferences (WC) with millimeter precision. After anthropometric measurements were taken, the BMI and waist to height ratio (WHtR) calculations were computed by the student researchers and verified by the researchers for accuracy.

\section{Data Analyses}

A series of two-way analysis of covariance (ANCOVA) were conducted to compare results of the administered instruments between ND and NND students, controlling for BMI. Further, a series of Spearman and Pearson correlation coefficients were computed to assess the relationship between the administered instruments and WC, calculated BMI, and WHtR (Statistical Package for Social Science [SPSS] IBM Corp, 2017).

\section{Results}

Blinded contributed $(\mathrm{n}=153)$ participants who were mostly women (72\%) and predominantly within a healthy BMI range of 18.5 to $24.9(66 \%)$ compared to Blinded which contributed $(n=144)$ participants who were also mostly women $(84 \%)$ and mainly within a healthy BMI range $(55 \%)$. Using Pearson Chi-Square, the gender was significantly different between categories, $\mathrm{p}=0.009$, but the ND major is traditionally mostly female, thus it was expected. Blinded had a larger percent of participants who were categorized as overweight $(24 \%)$, obese $(17 \%)$, and morbidly obese $(<1 \%)$ compared to Blinded respectively $(19 \%, 10 \%, 0 \%)$, which using Pearson ChiSquare, was statistically different at $\mathrm{p}=0.031$. Blinded and Blinded did not differ significantly by major ND vs. NND. A total 21 students declined the anthropometry measures and completed the survey only $(\mathrm{ND}=9, \mathrm{NND}=12)$ (Table 1).

Two-way ANCOVA's were conducted to examine the prevalence and differences in the AFA, FPS, and BAS survey outcomes between groups, after controlling for the covariate BMI. AFA was not significantly different in NND vs. ND when controlling for BMI, F (1) $=0.063$, $P=0.80$. FPS was not significantly different in NND vs. ND when controlling for BMI, F (1) $=0.09, P=0.75$. BAS remained significantly different between NND and ND when controlling for BMI, F (1) $=6.59, P=0.011$. 
Table 1.

Demographic Characteristics by Majors

\begin{tabular}{|c|c|c|}
\hline Category & Nutrition and Dietetics Major $(\mathrm{n}=153)$ & $\begin{array}{l}\text { Non-Nutrition and Dietetics } \\
(\mathrm{n}=144)\end{array}$ \\
\hline School Site & $\begin{array}{l}\text { Fresno State }(n=83,54.3 \%) \\
\text { San Francisco }(n=70,45.7 \%)\end{array}$ & $\begin{array}{l}\text { Fresno State }(\mathrm{n}=74,51.4 \%) \\
\text { San Francisco State }(\mathrm{n}=70,48.6 \%)\end{array}$ \\
\hline Gender & $\begin{array}{l}\text { Male }(n=37,24 \%) \\
\text { Female }(n=110,72 \%) \\
\text { Other }(n=6,4 \%)\end{array}$ & $\begin{array}{l}\text { Male }(n=23,16 \%) \\
\text { Female }(n=120,84 \%) \\
\text { Other }(n=1,<1 \%)\end{array}$ \\
\hline BMI Averages** & Mean $=24.09(\operatorname{SD} 5.27)$ & $25.26(\mathrm{SD} 4.49)$ \\
\hline BMI Category* & $\begin{array}{l}\text { Underweight }(\mathrm{n}=5,3 \%) \\
\text { Healthy }(\mathrm{n}=95,66 \%) \\
\text { Overweight }(\mathrm{n}=28,19 \%) \\
\text { Obese }(\mathrm{n}=15,10 \%) \\
\text { Morbidly Obese }(\mathrm{n}=1,<1 \%)\end{array}$ & $\begin{array}{l}\text { Underweight }(n=4,3 \%) \\
\text { Healthy }(n=72,55 \%) \\
\text { Overweight }(n=32,24 \%) \\
\text { Obese }(n=22,17 \%) \\
\text { Morbidly Obese }(n=2,<1 \%)\end{array}$ \\
\hline WCcm** & Mean $=80.05(\mathrm{SD}=10.30)$ & Mean $=83.47(\mathrm{SD}=12.96)$ \\
\hline WHtR & Mean $=0.49(\mathrm{SD}=0.065)$ & Mean $=0.51(\mathrm{SD}=0.074)$ \\
\hline
\end{tabular}

$*$ Pearson Chi-Square $<0.05 \quad * *$ paired T-test $<0.05$

\section{Anti-Fat-Attitude Questionnaire and} Anthropometric Measures

A Spearman's rank-order correlation coefficient was computed to assess the relationship between AFA scores and participants BMI category. There was weak positive non-significant correlation between AFA and BMI category, $\mathrm{r}=0.12, \quad P=.84$. A Pearson's correlation coefficient was computed to assess the relationship between AFA scores and participants WC. There was weak negative non-significant correlation between AFA and $\mathrm{WC}, \mathrm{r}=-0.021, P$ $=0.73$. A Pearson's correlation coefficient was computed to assess the relationship between AFA scores and participants WHtR. There was a weak negative non-significant correlation between AFA and WHtR, $\mathrm{r}=-0.020, P=.74$.

\section{Fat Phobia Scale and Anthropometric Measures}

A Spearman's rank-order correlation coefficient was computed to assess the relationship between
FPS scores and participants BMI category. There was weak positive significant correlation

between FPS and BMI category, $\mathrm{r}=0.168, P$ $=.005$. A Pearson's correlation coefficient was computed to assess the relationship between FPS scores and participants WC. There was weak positive significant correlation between FPS and $\mathrm{WCcm}, \mathrm{r}=.126, P=0.04$. A Pearson's correlation coefficient was computed to assess the relationship between FPS scores and participants WHtR. There was a weak positive significant correlation between FPS and WHtR, $\mathrm{r}=115, P$ $=.06$.

\section{Body Appreciation Scale and Anthropometric Measures}

A Spearman's rank-order correlation coefficient was computed to assess the relationship between BAS scores and participants BMI category. There was weak positive non-significant correlation between BAS and BMI category, $\mathrm{r}=0.047, P$ $=.44$. A Pearson's correlation coefficient was computed to assess the relationship between BAS 
scores and participants WC. There was weak positive non-significant correlation between BAS and WCcm, $\mathrm{r}=.104, P=0.09$. A Pearson's correlation coefficient was computed to assess the relationship between BAS scores and participants WHtR. There was a weak positive non-significant correlation between BAS and WHtR, $\mathrm{r}=0.079, P=.19$.

\section{Discussion}

The primary outcomes from this study indicated no statistically significant differences in AFA or the FPS between ND and NND, though higher average scores were observed in ND for both surveys (Table 2), indicating a greater trend towards anti-fat attitudes and fat phobia beliefs in ND majors. Interestingly, NND majors had a statistically significant higher BAS score compared to ND (Table 2), even with a higher prevalence of BMI scores representing overweight and obesity, and WHtR values $(\geq$ 0.50) (Table 1) (Ashwell \& Gibson, 2016; CDC, 2020). These results parallel other research studies in which ND students had higher prevalence of anti-fat attitudes and fat phobia scores; even when BMI's were categorized as healthy Nutrition and Dietetics students have been documented to have altered relationships with weight and dietary intake, with signs and symptoms of disordered eating and Diagnostic and Statistical Manual of Mental Disorders (DSM) defined eating disorders (Centers for Disease Control [CDC], 2020; Kolka \& Abayomi, 2012; Panza et al., 2018; Puhl et al., 2015; Swift et al., 2013). Therefore, understanding the prevalence of biases attitudes towards persons of overweight and obese is important to allow planning for appropriate methods of education and training in future RDNs of how to host discourse with patients to understand what the true etiology is related to their signs and symptoms of health risk.

To understand if a relationship existed between anthropometry and our survey tools, we analyzed and identified a series of weak positive statistically significant correlations between FPS scores and BMI indicating that as BMI increases, fat phobia increases, $\mathrm{r}=0.168, P=.005$. This same positive weak correlation was observed with $\mathrm{WC}$, $\mathrm{r}=.126, P=0.04$ and for WHtR at $\mathrm{r}=.115, P=.06$, also suggesting those with more adipose tissue around the central cavity had a higher fat phobias score. No significant correlations existed between AFA or BA and anthropometry.

The relationship between lifestyle habits and anthropometry has been well documented in medical professionals (Geller \& Watkins, 2018). Doctors have been recorded to engage in stigmatizing behaviors (e.g. recommending a diet even if the patient did not intend to discuss weight or assuming overeating behaviors because patient is overweight) and practice behaviors such as these have been associated with negative treatment outcomes (Remmert, Convertine, Roberts, Godfrey, \& Butryn, 2019). Evidence based reasoning for why medical professionals may act in this way has been linked to common training pedagogies such as the medical model or the public health model (Adler \& Stewart, 2009).

Table 2.

Anti-fat Attitudes, Fat Phobia Scale, and Body Appreciation Scale Differences in Non-nutrition/dietetics and Nutrition/Dietetics Students

\begin{tabular}{llllllll}
\hline Survey & Major & $\mathrm{N}$ & Mean & STD & SEM & t & $P$-value \\
\hline AFA & ND & 152 & $\mathbf{5 . 4 2}$ & 1.44 & .117 & -.291 & .771 \\
& NND & 142 & 5.37 & 1.47 & .123 & & \\
FPS & ND & 149 & $\mathbf{2 . 9 7}$ & .406 & .033 & -.302 & .763 \\
& NND & 143 & 2.95 & .352 & .029 & & \\
\multirow{2}{*}{ BAS } & ND & 150 & 3.61 & .663 & .054 & 2.49 & $\mathbf{0 . 0 1 3 *}$ \\
& NND & 140 & $\mathbf{3 . 8 1}$ & .702 & .059 & & \\
*Independent t-test $P<0.05$ & & & & & & & \\
\hline
\end{tabular}


Evidence based reasoning for why medical professionals may act in this way has been linked to common training pedagogies such as the medical model or the public health model (Adler $\&$ Stewart, 2009). The medical model focuses on training health practitioners to identify signs and symptoms related to disease risks, then recommend a treatment. Often the root cause of these signs and symptoms are connected to individual behaviors before genetics and biology, and usually without the consideration of external factors. Thus, when a person fails to follow the assigned treatment, the individual is blamed for lack of adherence. The public health model incorporates the idea of societal influences, environmental barriers and supports, and individual resource allocation (e.g. food security provisions). The RDN is trained predominantly using the medical model, therefore use of anthropometry, biochemical values, clinical signs and symptoms, and dietary history assists in diagnosing a nutrition related reason to support treatment recommendations. This approach, lacking in understanding the entire patient, is linked to weight bias and weight bias is linked to poor patient outcomes (Adler \& Stewart, 2009).

Overall, both groups had elevated anti-fat and fat phobia beliefs, with the ND values being nonsignificantly more elevated. While this weight bias and stigma are not isolated in nutrition and dietetics professionals, it is not systematically addressed in undergraduate or dietetic internship training. This deficiency in training can create barriers for patients to receive unbiased care if expectations are premeditated and may be an issue if patients do not have societal support for the treatment plans recommended, as the United States has been defined as an obesogenic environment, and, this can lead to perpetual failures in obesity treatment and prevention.

\section{Limitations}

A limitation to this study was lack of race/ethnicity information as well as specific major classification for the NND collected from both school sites. As noted in the literature review, stigma exists in other majors focusing on medical training (e.g. kinesiology, pre-nursing, nursing, health education, gerontology, and foods and community nutrition). In review of the data, it was observed that 41 of the $70 \mathrm{NND}$ at one school site were from health-related majors and this could have impacted our overall data of health professionals vs. non health professionals; however, we were able to analyze ND vs. nonNND. This information could possibly provide additional understanding of where weight bias concentrations exist for future interventions and education.

\section{Conclusions}

While dietary intake and lifestyle patterns do have associations with disease morbidity and long-term mortality, the importance of unbiased assessment is essential to facilitating patient led change (Resnicow, McMaster, Bocian, Harris, Zhou, Snetselaar, \& Wasserman, R. C., 2015). Current RDN nutrition education practice is focused on the medical model approach, which includes ample discussion about weight, BMI, calories, and their relationship with disease risk often in that order, but excludes inquiry about trauma, food access, and other experiences which may play a role in patient health. Weight stigma is an impediment to patient trust and permanent lifestyle change (Panza et al., 2018; Phelan et al., 2015; Puhl et al., 2009; Schwartz et al., 2003; Wu $\&$ Berry, 2018). The identification of weight stigma in novice nutrition and students, as seen in our study, highlights a training need for this population who are on their path to becoming RDNs. We did see a relationship between measures of body fat and also body appreciation, and this highlights that weight bias towards others might exist in our nutrition and dietetics students while they also have a high body appreciation. Building on this self-appreciation, a focus on empathy could be helpful in addition to changing the bigger picture. It is important to consider instilling a systematic and nationwide understanding of intuitive eating and body positive approaches to nutrition and health through the undergraduate curriculum by adding 
to the student learning outcomes in the ACEND accreditation requirements. This could assist in managing anti-fat attitude, fat phobia and lower body appreciation in nutrition and dietetics students (Bombak, 2014; Bombak, Monaghan, \& Rich, 2019). Future research should expand beyond a cross-sectional assessment of weight bias prevalence in nutrition and dietetics students, and study the implementation of existing weight bias curriculum into undergraduate training and /or investigate and test the development of a concise curriculum through interdisciplinary case studies, problem-based modules to delve deeper in to how to talk to the obese patient (UConn Rudd Center for Food Policy and Obesity, 2020). A deeper practice-based skill set to discuss succinctly not only weight, but the relationship to lifestyle, biological, socioeconomic, cultural, and social influences is essential to prevent the cycle of weight stigma (Tomiyama, Carr, Granberg, Major, Robinson, Sutin, \& Brewis, 2018).

\section{References}

Aboueid, S., Pouliot, C., Nur, T., Bourgeault, I., \& Giroux, I. (2019). Dietitians' perspectives on patient barriers and enablers to weight management: An application of the social-ecological model. Nutrition \& Dietetics: The Journal of the Dietitians Associated of Australia. 76(3), 353-362. doi:10.1111/1747-0080.12510

Adler, N. E., \& Stewart, J. (2009). Reducing obesity: motivating action while not blaming the victim. The Milbank Quarterly, 87(1), 49-70. doi: 10.1111/j.1468-0009.2009.00547.x

Andersen, D., Baird, S., Bates, T., Chapel, D.L., Cline, A.D., Ganesh, S.N... McCauley, S.M. (2018). Academy of Nutrition and Dietetics: Revised 2017 scope of practice for the registered dietitian nutritionist. Journal of the Academy of Nutrition and Dietetics, 118(1), 141-165. doi:10.1016/j.jand.2017.10.002

Anthropometry Procedures Manual (2017). Retrieved October 20, 2020, from Centers for Disease Control and Prevention (CDC), https://wwwn.cdc.gov/nchs/nhanes/continuousnhanes/manuals.aspx?BeginYear=2017

Ashwell, M., \& Gibson, S. (2016). Waist-to-height ratio as an indicator of 'early health risk': Simpler and more predictive than using a 'matrix' based on BMI and waist circumference. British Medical Journal Open, 6(3), 101-59. doi: 10.1136/bmjopen-2015-010159

Avalon, L.A., Tylka, T.L., \& Wood-Barcalow, N. (2005). The body appreciation scale: Development and psychometric evaluation. Body Image, 2(3), 285-297. doi: 10.1016/j.bodyim.2005.06.002.

Bacon, J., Scheltema, K., \& Robinson, B. (2001). Fat phobia scale revisited: The short form. International Journal of Obesity, 25(2), 252-257. doi: 10.1038/sj.ijo.0801537

Bégin, C., Carbonneau, E., Gagnon-Girouard, M.-P., Mongeau, L., Paquette, M.-C., Turcotte, M., \& Provencher, V. (2019). Eating-related and psychological outcomes of health at every size intervention in health and social services centers across the province of Québec. American Journal of Health Promotion, 33(2), 248-258. doi: 10.1177/0890117118786326

Bjornsson, A. S., Didie, E. R., \& Phillips, K. A. (2010). Body dysmorphic disorder. Dialogues in Clinical Neuroscience, 12(2), 221-232. doi: 10.31887/DCNS.2010.12.2/abjornsson.

Bombak, A., Monaghan, L.F., \& Rich, E. (2019). Dietary approaches to weight-loss, health at every size and beyond: Rethinking the war on obesity. Social Theory and Health, 17(1), 89-108. doi: 10.1057/s41285-018-0070-9

Bombak, A. (2014). Obesity, health at every size, and public health policy. American Journal of Public Health, 104(2), 60-67. doi: 10.2105/AJPH.2013.301486

Brown, L. B. (2009). Teaching the 'health at every size' paradigm benefits future fitness and health professionals. Journal of Nutrition Education and Behavior, 41(2), 144-145. doi: 10.1016/j.jneb.2008.04.358 
Constance, A., \& Sauter, C. (2011). Inspiring and supporting behavior change: A food and nutrition professional's counseling guide (1st ed.). Chicago: American Dietetic Association. website, https://www.worldcat.org/title/inspiring-and-supporting-behavior-change-a-food-nutrition-andhealth-professionals-counseling-guide/oclc/1015857806\&referer=brief results

Crandall, C. S. (1994). Prejudice against fat people: Ideology and self-interest. Journal of Personality and Social Psychology, 66(5), 882-894. doi: 10.1037/0022-3514.66.5.882

Defining overweight and obesity. (2020). Retrieved October 20, 2020, from Centers for Disease Control website, https://www.cdc.gov/obesity/adult/defining.html

Dennett, C. (2019). Weight bias in dietetics education. Today's Dietitian, 21(3), 36-42. website, https://www.todaysdietitian.com/newarchives/0319p36.shtml

Dwyer, J.J.M., Starr, A., Mills, C., \& Haines, J. (2016). Undergraduate, female, nutrition students' perceptions of curricular influence on attitudes toward individuals with obesity. Canadian Journal of Dietetic Practice and Research, 77(4), 177-182. doi: 10.3148/cjdpr-2016-010

FitzGerald, C., \& Hurst, S. (2017). Implicit bias in healthcare professionals: a systematic review. BMC Medical Ethics, 18(19), 1-16. doi: 10.1186/s12910-017-0179-8

Geller, G., \& Watkins, P.A. (2018). Addressing medical students' negative bias toward patients with obesity through ethics education. America Medical Association Journal of Ethics, 20(10), 948959. doi: 10.1001/amajethics.2018.948.

Greenhalgh, S. (2012). Weighty subjects: The biopolitics of the U.S. war on fat. American Ethnologist, 39(3), 471-487. doi:10.1111/j.1548-1425.2012.01375

González-Muniesa, P., Mártinez-González, M., Hu, F., Després, J.P., Matsuzama, Y., Loos, R.J.F... \& Martinez, J.A. (2017). Obesity. Nature Reviews Disease Primers 3, 17034(2017). doi: $\underline{10.1038 / \text { nrdp.2017.34 }}$

Hales, C.M., Carroll, M.D., Fryar, C.D., \& Ogden, C.L. (2017). Prevalence of obesity and severe obesity among adults: United States, 2017-2018. National Center of Health Statistics Data Briefs, 288(1), 1-8. website, https://www.cdc.gov/nchs/products/databriefs/db360.htm.

Jung, F.U.C.E., Luck-Sikorski, C., Wiemers, N., \& Riedel-Heller, S.G. (2015). Dietitians and nutritionists: Stigma in the context of obesity: A systematic review. Public Library of Science ONE, 10(10), 1-12. doi: 10.1371/journal.pone.0140276

Kolka, M., \& Abayomi, J.J.A. (2012). Body image dissatisfaction among food-related degree students. Nutrition \& Food Science, 42(3), 139-147. doi: 10.1108/00346651211228423

O’Brien, K.S., Puhl, R.M., Latner, J.D., Mir, A.S., \& Hunter, J.A. (2010). Reducing anti-fat prejudice in preservice health students: A randomized trial. Obesity, 18(11), 2138-2144. doi:10.1038/oby. 2010.79

Obara, A.A., Vivolo, S.R.G.F., \& Alvarenga, M.S. (2018). Preconceito relacionado ao peso na conduta nutricional: um estudo com estudantes denutrição. Cadernos de Saúde Pública, 34(8), 1-16. doi: 10.1590/0102-311x00088017

Ogden, C. L., Fryar, C. D., Hales, C. M., Carroll, M. D., Aoki, Y., \& Freedman, D. S. (2018). Differences in obesity prevalence by demographics and urbanization in U.S. children and adolescents, 20132016. Journal of the American Medical Association, 319(23), 2410-2418. doi: $10.1001 /$ jama.2018.5158

Panza, G. A., Armstrong, L. E., Taylor, B. A., Puhl, R. M., Livingston, J., \& Pescatello, L. S. (2018). Weight bias among exercise and nutrition professionals: A systematic review: Weight bias in exercise and nutrition. Obesity Reviews, 19(11), 1492-1503. doi: 10.1111/obr.12743

Pearl, R. L., \& Puhl, R. M. (2016). The distinct effects of internalizing weight bias: An experimental study. Body Image, 17(1), 38-42. doi: 10.1016/j.bodyim.2016.02.002 
Pearl, R. L., Wadden, T. A., Tronieri, J. S., Chao, A. M., Alamuddin, N., Bakizada, Z. M., .. . \& Berkowitz, R. I. (2018). Sociocultural and familial factors associated with weight bias internalization. Obesity Facts, 11(2), 157-164. doi: 10.1159/000488534

Phelan, S., Burgess, D., Yeazel, M., Hellerstedt, W., Griffin, J., \& Ryn, M.V. (2015). Impact of weight bias and stigma on quality of care and outcomes for patients with obesity. Obesity Reviews, 16(1), 319-326. doi: 10.1111/obr.12266.

Puhl, R., Wharton, C., \& Heuer, C. (2009). Weight bias among dietetics students: Implications for treatment practices. Journal of the American Dietetic Association, 109(3), 438-444. doi: 10.1016/i.jada.2008.11.034

Puhl, R.M., Latner, J. D., O’brien, K. S., Luedicke, J., Danielsdottir, S., \& Forhan, M. (2015). A multinational examination of weight bias: Predictors of anti-fat attitudes across four countries. International Journal of Obesity, 39(7), 1166-1173. doi: 10.1038/ijo.2015.32.

Raynor, H.A., \& Champagne, C.M. (2016). Position of the Academy of Nutrition and Dietetics: Interventions for the treatment of overweight and obesity in adults. Journal of the Academy of Nutrition and Dietetics. 116(1), 129-147. doi: 10.1016/j.jand.2015.10.031.

Remmert, J. E., Convertino, A. D., Roberts, S. R., Godfrey, K. M., \& Butryn, M. L. (2019). Stigmatizing weight experiences in health care: Associations with BMI and eating behaviours. Obesity Science and Practice, 5(6), 555-563. doi: 10.1002/osp4.379

Resnicow, K., McMaster, F., Bocian, A., Harris, D., Zhou, Y., Snetselaar, L., Schwartz, R., Myers, E., Gotlieb, J., Foster, J., Hollinger, D., Smith, K., Woolford, S., Mueller, D., \& Wasserman, R. C. (2015). Motivational interviewing and dietary counseling for obesity in primary care: An RCT. Pediatrics, 135(4), 649-657. https://doi.org/10.1542/peds.2014-1880

Ross, R., Neeland, I. J., Yamashita, S., Shai, I., Seidell, J., Magni, P., Santos, R. D., Arsenault, B., Cuevas, A., Hu, F. B., Griffin, B. A., Zambon, A., Barter, P., Fruchart, J. C., Eckel, R. H., Matsuzawa, Y., \& Després, J. P. (2020). Waist circumference as a vital sign in clinical practice: A Consensus Statement from the IAS and ICCR Working Group on Visceral Obesity. Nature Reviews Endocrinology, 16(3), 177-189. https://doi.org/10.1038/s41574-019-0310-7

Schvey, N. A., Puhl, R. M., \& Brownell, K. D. (2014). The stress of stigma: Exploring the effect of weight stigma on cortisol reactivity. Psychosomatic Medicine, 76(1), 156-62. doi:10.1097/PSY.0000000000000031

Schwartz, M. B., O’Neal, H., Chambliss, K., Brownell, K. D., Blair, S. N., \& Billington, C. (2003). Weight bias among health professionals specializing in obesity. Obesity Research, 11(1), 10331039. doi:10.1038/oby.2003.142

Statistical Package for Social Science (SPSS) IBM Corp. (2017). IBM SPSS statistics for windows (Version 25.0) [Computer software]. Armonk, NY: IBM Corp.

Swift, J. A., Hanlon, S., El-Redy, L., Puhl, R.M., \& Glazebrook, C. (2013). Weight bias among UK trainee dietitians, doctors, nurses and nutritionists. Journal of Human Nutrition and Dietetics, 26(4), 395-402. doi: 10.1111/jhn.12019.

Swift, J. A., Tischler, V., Markham, S., Gunning, I., Glazebrook, C., Beer, C., \& Puhl, R. (2013). Are anti-stigma films a useful strategy for reducing weight bias among trainee healthcare professionals? Results of a pilot randomized control trial. Obesity Facts, 6(1), 91-102. doi: $10.1159 / 000348714$.

Teachman, B.A., \& Brownell, K.D. (2001). Implicit anti-fat bias among health professionals: Is anyone immune? International Journal of Obesity, 25(10), 15-25. doi: 10.1038/sj.ijo.0801745

Tomiyama, A., Carr, D., Granberg, E. M., Major, B., Robinson, E., Sutin, A. R., \& Brewis, A. (2018). How and why weight stigma drives the obesity 'epidemic' and harms health. BMC Medicine, 16(123), 1-12. doi: 10.1186/s12916-018-1116-5 
Tremelling, K., Sandon, L., Vega, G.L., McAdams, C.J. (2017). Orthorexia Nervosa and eating disorder symptoms in registered dietitian nutritionists in the United States. Journal of the Academy of Nutrition and Dietetics, 2117(10), 1612-1617. doi: 10.1016/j.jand.2017.05.001.

Weight bias and stigma. (2020). Retrieved October 20, 2020, from UConn Rudd Center for Food Policy and Obesity website, http://uconnruddcenter.org/weight-bias-stigma

Wu, Y-K., Berry, D.C. (2018). Impact of weight stigma on physiological and psychological health outcomes for overweight and obese adults: A systematic review. Journal of Advanced Nursing, 74(5), 1030-1042. doi:10.1111/jan.13511

Corresponding Author Information Lisa Herzig, PhD, RDN, CDCES,

Fresno State University, 5300 North Campus Drive M/S FF17

Fresno California, CA 93740

Number: 559-278-2043

Fax: 559-278-4496

lherzig@csufresno.edu 